\title{
Spanning Trees and a Conjecture of Kontsevich
}

\author{
Richard P. Stanley* \\ Department of Mathematics, Massachusetts Institute of Technology, Cambridge, MA 02139, \\ USA \\ rstan@math.mit.edu
}

Received October 30, 1998

AMS Subject Classification: 05E99

\begin{abstract}
Kontsevich conjectured that the number of zeros over the field $\mathbb{F}_{q}$ of a certain polynomial $Q_{G}$ associated with the spanning trees of a graph $G$ is a polynomial function of $q$. We show the connection between this conjecture, the Matrix-Tree Theorem, and orthogonal geometry. We verify the conjecture in certain cases, such as the complete graph, and discuss some modifications and extensions.
\end{abstract}

Keywords: spanning tree, Matrix-Tree Theorem, orthogonal geometry

\section{Introduction}

Let $G$ be a (finite) graph with vertex set $V(G)=\left\{v_{1}, \ldots, v_{n}\right\}$ and edge set $E(G)=$ $\left\{e_{1}, \ldots, e_{s}\right\}$. For now we allow loops (edges from a vertex to itself) and multiple edges. For each edge $e$ of $G$, associate an indeterminate $x_{e}$. If $S \subseteq E(G)$, let

$$
x^{S}=\prod_{e \in S} x_{e} .
$$

Let $T$ be a spanning tree of $G$ and let $T^{\prime}$ denote the (edge) complement of $T$. Define polynomials $P_{G}(x)$ and $Q_{G}(x)$ in the variables $x=\left(x_{e}\right)_{e \in E(G)}$ by

$$
\begin{aligned}
P_{G}(x) & =\sum_{T} x^{T} \\
Q_{G}(x) & =\sum_{T} x^{T},
\end{aligned}
$$

where both sums range over all spanning trees $T$ of $G$. For instance, if $G$ is a four-cycle with edge set $\{1,2,3,4\}$, then

$$
\begin{aligned}
P_{G}(x) & =x_{1}+x_{2}+x_{3}+x_{4} \\
Q_{G}(x) & =x_{1} x_{2} x_{3}+x_{1} x_{2} x_{4}+x_{1} x_{3} x_{4}+x_{2} x_{3} x_{4} .
\end{aligned}
$$

* Partially supported by NSF grant \#DMS-9743966. 
Note that if $G$ is not connected, then $P_{G}(x)=Q_{G}(x)=0$. Note also that for any $G$, we have

$$
Q_{G}(x)=\left(\prod_{e \in E(G)} x_{e}\right) P_{G}(1 / x),
$$

where $P_{G}(1 / x)$ denotes the result of substituting $1 / x_{e}$ for $x_{e}$ in $P_{G}(x)$ (for all $e$ ).

Let $q$ be a prime power, and let $f_{G}(q)$ (respectively, $g_{G}(q)$ ) denote the number of ways of substituting elements of the finite field $\mathbb{F}_{q}$ for the variables $x_{e}$ so that $P_{G}(x) \neq 0$ (respectively, $Q_{G}(x) \neq 0$ ). For instance, if $G$ is a four-cycle as above and we want $P_{G}(x) \neq 0$, then $x_{1}, x_{2}, x_{3}$ can be arbitrary; then there are $q-1$ choices for $x_{4}$. Hence, $f_{G}(q)=q^{3}(q-1)$. If we want $Q_{G}(x) \neq 0$, then it takes a little more work to see that $g_{G}(q)=q(q-1)\left(q^{2}-2\right)$. More generally, if $C_{n}$ denotes an $n$-cycle, then it is not hard to check (as was done in the case of $g_{C_{n}}(q)$ by J. Stembridge) that

$$
\begin{aligned}
f_{C_{n}}(q)= & q^{n-1}(q-1) \\
g_{C_{n}}(q)= & n(q-1)^{n-1}+(q-1)^{n}-(q-1)^{n-1}+(q-1)^{n-2} \\
& +\cdots+(-1)^{n-1}(q-1) .
\end{aligned}
$$

Note that if $G$ is not connected, then $f_{G}(q)=g_{G}(q)=0$, since $P_{G}(x)=Q_{G}(x)=0$.

In a lecture delivered at the Rutgers University Gelfand Seminar on December 8, 1997, M. Kontsevich stated the conjecture, in connection with the evaluation of certain integrals appearing in perturbative quantum field theory, that $f_{G}(q)$ is a "universal polynomial" in $q$, i.e., a polynomial in $q$ independent of the characteristic $p$ of the field $\mathbb{F}_{q}$. We have been unable to resolve Kontsevich's conjecture, but in Sections 4 and 5 we present evidence in its favor while in Section 6 we present evidence against it. Let us mention that John Stembridge [9] has verified that $g_{G}(q)$ is a polynomial for all graphs with at most twelve edges.

\section{Some General Observations}

Given the graph $G$, let $S$ be a subset of $E(G)$. Define $f_{G, S}(q)$ (respectively, $f_{G, S}^{+}(q)$ ) to be the number of ways of substituting elements of $\mathbb{F}_{q}$ for the variables $x_{e}$ such that $x_{e}=0$ if $e \in S$ (respectively, if and only if $e \in S$ ) so that $P_{G}(x) \neq 0$. Similarly, define $g_{G, S}(q)$ and $g_{G, S}^{+}(q)$ using $Q_{G}(x)$. In particular, $f_{G, \emptyset}(q)=f_{G}(q)$ and $g_{G, \emptyset}(q)=g_{G}(q)$. Now,

$$
\begin{aligned}
& f_{G, S}(q)=\sum_{T \supseteq S} f_{G, T}^{+}(q) \\
& g_{G, S}(q)=\sum_{T \supseteq S} g_{G, T}^{+}(q) .
\end{aligned}
$$

Hence, by the Principle of Inclusion-Exclusion, we have

$$
\begin{aligned}
& f_{G, S}^{+}(q)=\sum_{T \supseteq S}(-1)^{\#(T-S)} f_{G, T}(q) \\
& g_{G, S}^{+}(q)=\sum_{T \supseteq S}(-1)^{\#(T-S)} g_{G, T}(q) .
\end{aligned}
$$


Now, note that $g_{G, S}(q)=g_{H}(q)$, where $H$ is the spanning subgraph of $G$ with edge set $E(G)-S$. Similarly, if $S$ is acyclic (contains no cycle), then $f_{G, S}(q)=f_{K}(q)$ where $K$ denotes $G$ with the edges in $S$ contracted to points. On the other hand, if $S$ contains a cycle, then $f_{G, S}(q)=0$. Finally, observe from Equation (1.2) that for any graph $H$ we have

$$
f_{H, \emptyset}^{+}(q)=g_{H, \emptyset}^{+}(q) .
$$

From these observations we obtain the following result.

Proposition 2.1. Let $n \geq 1$. Then $f_{G}(q)$ is a universal polynomial in $q$ for all graphs $G$ on at most $n$ vertices if and only if the same is true for $g_{G}(q)$.

From now on we will deal only with $Q_{G}(x)$ and $g_{G}(q)$. Note also that if $G^{\prime}$ denotes $G$ with either one new loop added to a vertex or one edge replaced by two edges, then

$$
g_{G^{\prime}}(q)=q \cdot g_{G}(q) .
$$

Hence it suffices to assume from now on that $G$ is simple, i.e., has no loops or multiple edges.

John Stembridge has pointed out that a minimal counterexample to Kontsevich's conjecture must have edge-connectivity at least three, i.e, the graph cannot be disconnected by the removal of two edges. Indeed, suppose there are two edges $e$ and $e^{\prime}$ whose removal disconnects $G$, leaving the disjoint union of $G_{1}$ and $G_{2}$. Let $G^{\prime}$ denote the graph obtained from $G$ by contracting $e$, and let $G^{\prime \prime}$ be obtained by further contracting $e^{\prime}$. Then it is easy to check that

$$
g_{G}(q)=q \cdot g_{G_{1}}(q) g_{G_{2}}(q)+(q-2) g_{G^{\prime}}(q)+(q-1) g_{G^{\prime \prime}}(q) .
$$

As a final remark, recall that by the rationality of the zeta function of an algebraic variety over a finite field (due to Dwork [5]), for a fixed prime power $q$, there exist algebraic integers $\alpha_{1}, \ldots, \alpha_{r}$ and $\beta_{1}, \ldots, \beta_{s}$ such that for all $m \geq 1$,

$$
g_{G}\left(q^{m}\right)=\sum \alpha_{i}^{m}-\sum \beta_{j}^{m} .
$$

Since the $\alpha_{i}$ 's and $\beta_{j}$ 's are algebraic integers, it is easy to deduce the following consequence.

Proposition 2.2. If $g_{G}(q)$ is a polynomial in $q$ with rational coefficients, then in fact $g_{G}(q)$ has integer coefficients.

\section{The Matrix-Tree Theorem and Nonsingular Symmetric Matrices}

The fundamental tool for our results is the Matrix-Tree Theorem. This result was stated by J. J. Sylvester in 1857. The first proof was published by C. W. Borchardt in 1860 (though G. Kirchhoff actually proved the theorem in a dual form in 1847). The MatrixTree Theorem is often attributed to A. Cayley because he cited Sylvester's work in 1856 before it was published. For an exposition of the Matrix-Tree Theorem and more precise references, see Chapter 5.6 and the Notes to Chapter 5 of [8]. Let $G$ be a graph without loops or multiple edges on the vertex set $\left\{v_{1}, \ldots, v_{n}\right\}$, and as above associate 
the indeterminate $x_{e}$ with every edge $e$ of $G$. Let $L=L(G)=\left(L_{i j}\right)_{1}^{n}$ be the $n \times n$ matrix defined by

$$
L_{i j}= \begin{cases}-x_{e}, & \text { if } i \neq j \text { and } e \text { has vertices } v_{i} \text { and } v_{j}, \\ 0, & \text { if } i \neq j \text { and there is no edge with vertices } v_{i} \text { and } v_{j}, \\ \sum_{e} x_{e}, & \text { if } i=j \text { and } e \text { ranges over all edges incident to } v_{i} .\end{cases}
$$

Let $L_{0}=L_{0}(G)$ denote $L$ with the last row and column removed. We call $L$ the generic Laplacian matrix of $G$, and $L_{0}$ the reduced generic Laplacian matrix of $G$.

Theorem 3.1. (The Matrix-Tree Theorem) We have

$$
Q_{G}(x)=\operatorname{det} L_{0}(G)
$$

Corollary 3.2. Let $L_{0}$ be the reduced generic Laplacian matrix of the connected graph $G$. Then $g_{G}(q)$ is the number of solutions to $\operatorname{det} L_{0} \neq 0$ over $\mathbb{F}_{q}$.

We say that a vertex $v$ of the simple graph $G$ is an apex if $v$ is incident to every other vertex of $G$. For graphs with apexes, Corollary 3.2 has the following variant.

Theorem 3.3. Let $G$ be a simple graph with vertices $v_{1}, \ldots, v_{n}$ such that $v_{n}$ is an apex. Then $g_{G}(q)$ is equal to the number of $(n-1) \times(n-1)$ nonsingular symmetric matrices $M$ over $\mathbb{F}_{q}$ such that $M_{i j}=0$ whenever $i \neq j$ and $G$ has no edge between $v_{i}$ and $v_{j}$.

Proof. Let $e_{i}$ be the unique edge of $G$ with vertices $v_{i}$ and $v_{n}$, for $1 \leq i \leq n-1$. The $(i, i)$-entry $\left(L_{0}\right)_{i i}$ of the reduced generic Laplacian matrix $L_{0}$ has the form $x_{e_{i}}+$ other terms, and nowhere else does $x_{e_{i}}$ appear in $L_{0}$. Hence, we can replace $\left(L_{0}\right)_{i i}$ with $x_{e_{i}}$ without affecting the set of $q^{\# E}$ matrices we obtain from $L_{0}$ by letting the $x_{e}$ 's assume all possible values in $\mathbb{F}_{q}$. Similarly we do not affect this set by changing the signs of the off-diagonal entries. But then $L_{0}$ becomes a symmetric matrix $L_{0}^{*}$ whose entries are generic except that $\left(L_{0}^{*}\right)_{i j}=0$ whenever $i \neq j$ and $G$ has no edge between $v_{i}$ and $v_{j}$, and the proof follows.

\section{The Complete Graph}

Theorem 3.3 allows us to evaluate $g_{G}(q)$ explicitly for certain graphs $G$. We first consider the complete graph $K_{n}$, with $n$ vertices and one edge between every pair of distinct vertices. Hence, by Theorem 3.3, $g_{K_{n}}(q)$ is just the total number of nonsingular $(n-1) \times(n-1)$ symmetric matrices over $\mathbb{F}_{q}$. This number was first computed for $q$ odd by L. Carlitz [2, Theorem 3] as part of a much more general result. A simpler proof valid for any $q$ was later given by J. MacWilliams [6, Theorem 2]. We will sketch the proof of MacWilliams and a second proof based on orthogonal geometry over $\mathbb{F}_{q}$, since both proofs will lead to generalizations.

Theorem 4.1. We have

$$
g_{K_{n}}(q)= \begin{cases}q^{m(m-1)}(q-1)\left(q^{3}-1\right) \cdots\left(q^{2 m-1}-1\right), & n=2 m, \\ q^{m(m+1)}(q-1)\left(q^{3}-1\right) \cdots\left(q^{2 m-1}-1\right), & n=2 m+1 .\end{cases}
$$


First Proof. (J. MacWilliams) It is more convenient to consider $g_{K_{n+1}}(q)$ rather than $g_{K_{n}}(q)$, so that we are enumerating $n \times n$ invertible symmetric matrices over $\mathbb{F}_{q}$. Let $h(n, r)$ denote the number of $n \times n$ symmetric matrices $M$ over $\mathbb{F}_{q}$ of rank $r$. We claim that

$$
h(n, r)= \begin{cases}\prod_{i=1}^{s} \frac{q^{2 i}}{q^{2 i}-1} \cdot \prod_{i=0}^{2 s-1}\left(q^{n-i}-1\right), & 0 \leq r=2 s \leq n, \\ \prod_{i=1}^{s} \frac{q^{2 i}}{q^{2 i}-1} \cdot \prod_{i=0}^{2 s}\left(q^{n-i}-1\right), & 0 \leq r=2 s+1 \leq n .\end{cases}
$$

An $(n+1) \times(n+1)$ symmetric matrix may be written as

$$
N=\left[\begin{array}{cc}
\beta & y \\
y^{t} & M
\end{array}\right]
$$

where $M$ is an $n \times n$ symmetric matrix, $\beta \in \mathbb{F}_{q}$, and $y \in \mathbb{F}_{q}^{n}$. Elementary linear algebra arguments (given explicitly in [6]) show that from a particular $M$ of rank $r$ we obtain:

- $q^{n+1}-q^{r+1}$ matrices $N$ of rank $r+2$,

- $(q-1) q^{r}$ matrices $N$ of rank $r+1$,

- $q^{r}$ matrices $N$ of rank $r$,

- no matrices of other ranks.

There follows the recurrence

$$
h(n+1, r)=q^{r} h(n, r)+(q-1) q^{r-1} h(n, r-1)+\left(q^{n+1}-q^{r-1}\right) h(n, r-2) .
$$

One can check that the solution to this recurrence satisfying the initial conditions $h(n, 0)$ $=1$ and $h(n, r)=0$ for $r>n$ is given by (4.2). The proof follows from the case $r=n$.

\section{Second Proof.}

Case 1. $q$ odd. Let $\operatorname{Sym}^{+}(n, q)$ (respectively, $\left.\operatorname{Sym}^{-}(n, q)\right)$ denote the set of all $n \times n$ nonsingular symmetric matrices over $\mathbb{F}_{q}$ whose determinant is a square (respectively, a nonsquare) in $\mathbb{F}_{q}$. Let $\Omega^{+}(n, q)$ denote the group of all matrices $A \in \operatorname{GL}(n, q)$ satisfying $A A^{t}=I$. (We will be dealing with various groups closely related to the orthogonal groups $O(n, q)$. We will use the notation $\Omega$ rather than $O$ to make clear that our groups are related but in general are not equal to the usual orthogonal groups.) By standard results concerning orthogonal geometry over a finite field (implicit in [4]), the map $f: \operatorname{GL}(n, q) \rightarrow \operatorname{Sym}^{+}(n, q)$ defined by $f(A)=A A^{t}$ is surjective, and $f^{-1}\left(A A^{t}\right)=A \cdot \Omega^{+}(n, q)$, the left coset of $\Omega^{+}(n, q)$ in $\operatorname{GL}(n, q)$ containing $A$. Hence, all fibers $f^{-1}(B)$ have cardinality $\# \Omega^{+}(n, q)$, so

$$
\# \operatorname{Sym}^{+}(n, q)=\frac{\# \mathrm{GL}(n, q)}{\# \Omega^{+}(n, q)} \text {. }
$$

Similarly, let $\alpha$ be a fixed nonsquare in $\mathbb{F}_{q}$, and let $\Omega^{-}(n, q)$ denote the group of all matrices $A \in \operatorname{GL}(n, q)$ satisfying $A D A^{t}=D$, where $D=\operatorname{diag}(\alpha, 1,1, \ldots, 1)$. Then the map 
$f: \operatorname{GL}(n, q) \rightarrow \operatorname{Sym}^{-}(n, q)$ defined by $f(A)=A D A^{t}$ is surjective, and $f^{-1}\left(A D A^{t}\right)=$ $A \cdot \Omega^{-}(n, q)$. Hence, all fibers $f^{-1}(B)$ have cardinality $\# \Omega^{-}(n, q)$, so

$$
\# \operatorname{Sym}^{-}(n, q)=\frac{\# \mathrm{GL}(n, q)}{\# \Omega^{-}(n, q)} .
$$

Since $\operatorname{Sym}(n, q)=\operatorname{Sym}^{+}(n, q) \cup \operatorname{Sym}^{-}(n, q)$, there follows

$$
\# \operatorname{Sym}(n, q)=\frac{\# \mathrm{GL}(n, q)}{\# \Omega^{+}(n, q)}+\frac{\# \mathrm{GL}(n, q)}{\# \Omega^{-}(n, q)} .
$$

The order of $\operatorname{GL}(n, q)$ is well known and easily seen to be

$$
\# \mathrm{GL}(n, q)=\left(q^{n}-1\right)\left(q^{n}-q\right) \cdots\left(q^{n}-q^{n-1}\right) .
$$

Moreover, the orders of $\Omega^{+}(n, q)$ and $\Omega^{-}(n, q)$ were computed by Dickson [4, Theorem 172] as follows:

$$
\begin{aligned}
& \# \Omega^{+}(n, q)= \begin{cases}2 q^{m(m-1)}\left(q^{m}-1\right) \prod_{i=1}^{m-1}\left(q^{2 i}-1\right), & n=2 m, q \equiv 1(\bmod 4), \\
2 q^{m(m-1)}\left(q^{m}-(-1)^{m}\right) \prod_{i=1}^{m-1}\left(q^{2 i}-1\right), & n=2 m, q \equiv 3(\bmod 4), \\
2 q^{m^{2}} \prod_{i=1}^{m}\left(q^{2 i}-1\right), & n=2 m+1 .\end{cases} \\
& \# \Omega^{-}(n, q)= \begin{cases}2 q^{m(m-1)}\left(q^{m}+1\right) \prod_{i=1}^{m-1}\left(q^{2 i}-1\right), & n=2 m, q \equiv 1(\bmod 4), \\
2 q^{m(m-1)}\left(q^{m}+(-1)^{m}\right) \prod_{i=1}^{m-1}\left(q^{2 i}-1\right), & n=2 m, q \equiv 3(\bmod 4), \\
2 q^{m^{2}} \prod_{i=1}^{m}\left(q^{2 i}-1\right), & n=2 m+1 .\end{cases}
\end{aligned}
$$

Substituting these numbers into Equation (4.4) (after replacing $n$ by $n-1$ ) yields (4.1) (when $q$ is odd).

Case 2. $q$ even. This case is analogous to the odd case, but the details are somewhat different. When $n$ is odd, it follows from [1, Theorem 7] that the map $f: \operatorname{GL}(n, q) \rightarrow$ $\operatorname{Sym}(n, q)$ defined by $f(A)=A A^{t}$ is surjective, with $\# f^{-1}\left(A A^{t}\right)=A \cdot \Omega(n, q)$ (where $\left.\Omega(n, q)=f^{-1}(I)\right)$. Hence,

$$
\# \operatorname{Sym}(n, q)=\frac{\# \mathrm{GL}(n, q)}{\# \Omega(n, q)} .
$$

Dickson [4, p. 206] showed that

$$
\# \Omega(2 m+1, q)=q^{m^{2}} \prod_{i=1}^{m}\left(q^{2 i}-1\right),
$$


so (4.1) follows in this case.

When $n=2 m$, let $\operatorname{Sym}^{+}(n, q)$ (respectively, $\left.\operatorname{Sym}^{-}(n, q)\right)$ denote the set of $n \times n$ nonsingular matrices over $\mathbb{F}_{q}$ with at least one nonzero entry on the main diagonal (respectively, with all 0's on the main diagonal). (When $n$ is odd, we have $\operatorname{Sym}^{-}(n, q)=$ $\emptyset$, since a symmetric matrix of odd order with zero diagonal over a field of characteristic two is singular.) It was shown by Albert [1, Thm. 7] that the map $f: \operatorname{GL}(n, q) \rightarrow$ $\operatorname{Sym}^{+}(n, q)$ defined by $f(A)=A A^{t}$ is surjective. Let $E$ be the direct sum of $m$ copies of the matrix $\left[\begin{array}{ll}0 & 1 \\ 1 & 0\end{array}\right]$. The map $f: \operatorname{GL}(n, q) \rightarrow \operatorname{Sym}^{-}(n, q)$ defined by $f(A)=A E A^{t}$ is surjective. If $\Omega^{-}(n, q)=f^{-1}(I)$, then $f^{-1}\left(A E A^{t}\right)=A \cdot \Omega^{-}(n, q)$. Hence reasoning as before gives

$$
\# \operatorname{Sym}(n, q)=\frac{\# \mathrm{GL}(n, q)}{\# \Omega^{+}(n, q)}+\frac{\# \mathrm{GL}(n, q)}{\# \Omega^{-}(n, q)} .
$$

It follows from the work of Dickson [4, Chapter VIII] that

$$
\begin{aligned}
& \# \Omega^{+}(2 m, q)=q^{m^{2}} \prod_{i=1}^{m-1}\left(q^{2 i}-1\right), \\
& \# \Omega^{-}(2 m, q)=q^{m^{2}} \prod_{i=1}^{m}\left(q^{2 i}-1\right),
\end{aligned}
$$

from which we obtain (4.1) in this final case.

Note that the first proof of Theorem 4.1 makes it clear from the start that $g_{K_{n}}(q)$ is a polynomial, while in the second proof (especially when $n$ is even), it appears somewhat miraculous that the computations in odd and even characteristics lead to the same final answer. The fact that the two cases yield the same answer boils down to the identity

$$
\frac{1}{2}\left(\frac{1}{q^{m}-1}+\frac{1}{q^{m}+1}\right)=\frac{1}{q^{m}}+\frac{1}{q^{m}\left(q^{2 m}-1\right)}
$$

\section{Some Generalizations of the Complete Graph}

The two proofs we gave for Theorem 4.1 can be extended to more general results. For the first generalization, let $G$ be an $n$-vertex graph (without loops or multiple edges). Let $L_{0}$ denote the reduced generic Laplacian matrix of $G$, with respect to some vertex $v$ indexing the last row and column of $L$. Write $h(G, r)$ for the number of ways of evaluating $L_{0}$ over $\mathbb{F}_{q}$ (i.e., the number of ways to substitute elements of $\mathbb{F}_{q}$ for the variables appearing in $L_{0}$ ) such that a matrix of rank $r$ is obtained. Thus, if $v$ is an apex of $G$, then by Theorem 3.3, we have $h(G, n-1)=g_{G}(q)$.

Theorem 5.1. Let $G$ be an $n$-vertex graph with an apex, and let $G^{*}$ denote $G$ with an apex adjoined (so $G^{*}$ has at least two apexes). Then

$$
h\left(G^{*}, r\right)=q^{r} h(G, r)+(q-1) q^{r-1} h(G, r-1)+\left(q^{n}-q^{r-1}\right) h(G, r-2) .
$$


Proof. The proof is essentially the same as the first proof of Theorem 4.1. Let the vertices of $G^{*}$ be $v_{1}, \ldots, v_{n+1}$, where $v_{1}$ and $v_{n+1}$ are apexes. Let $e_{i}$ denote the edge from $v_{1}$ to $v_{i}$ for $2 \leq i \leq n+1$, and write $x_{i}$ for $x_{e_{i}}$. We then have

$$
L_{0}\left(G^{*}\right)=\left[\begin{array}{cc}
\beta & y \\
y^{t} & L_{0}(G)
\end{array}\right],
$$

where $y=-\left(x_{2}, \ldots, x_{n}\right)$ and $\beta=x_{2}+\cdots+x_{n}+x_{n+1}$. Since $x_{n+1}$ appears in $L_{0}\left(G^{*}\right)$ only in the entry $\beta$, we may replace $\beta$ by $x_{n+1}$ without affecting the set of matrices we obtain from $L_{0}\left(G^{*}\right)$ by letting the $x_{e}$ 's assume all possible values in $\mathbb{F}_{q}$. Similarly, we may replace $y$ by $-y$. From a particular rank $r$ evaluation of $L_{0}(G)$ over $\mathbb{F}_{q}$, we can apply the reasoning in the first proof of Theorem 4.1 to get the recurrence (5.1).

Theorem 5.1 provides a simple recursive procedure for computing $g_{G}(q)$ for a graph with "few" missing edges (and hence many apexes). For instance, for $n>k$, let $K_{n}-K_{k}$ denote the complete graph $K_{n}$ on the vertex set $[n]=\{1,2 \ldots, n\}$ with all edges $i j$ removed where $i, j \in\{1,2, \ldots, k\}, i \neq j$. When $k=n-1, L_{0}\left(K_{n}-K_{k}\right)$ is just a generic diagonal matrix, so we get

$$
h\left(K_{n}-K_{n-1}, r\right)=\left(\begin{array}{c}
n-1 \\
r
\end{array}\right)(q-1)^{r} .
$$

Hence, in principle, Theorem 5.1 can be used iteratively to compute $h\left(K_{n}-K_{k}, r\right)$ for any $n, k, r$. In particular, it follows that $g_{K_{n}-K_{k}}(q) \in \mathbb{Z}[q]$, verifying Kontsevich's conjecture in this case. When $k=1$, we have $K_{n}-K_{1}=K_{n}$, which was dealt with in Theorem 4.1. When $k=2$, we have $K_{n}-K_{2}=K_{n}-e$, the complete graph $K_{n}$ with one edge removed. We compute $g_{K_{n}-e}(q)$ by another method in Theorem 5.4 (the case $s=1$ ). For $3 \leq k \leq 5$, we use Theorem 5.1 to produce the following conjecture. One could easily extend this conjecture to other small values of $k$, but what would be more interesting is a conjecture for general $n$ and $k$.

Conjecture 5.2. We have

$$
\begin{aligned}
g_{K_{2 m}-K_{3}}(q)= & q^{m(m-1)}(q-1)\left(q^{3}-1\right) \cdots\left(q^{2 m-5}-1\right) \\
& \cdot\left(q^{4 m-3}-4 q^{2 m-4}+3 q^{2 m-5}-q^{2 m-6}+1\right), m \geq 2, \\
g_{K_{2 m+1}-K_{3}}(q)= & q^{m^{2}+m-3}(q-1)\left(q^{3}-1\right) \cdots\left(q^{2 m-3}-1\right) \\
& \cdot\left(q^{2 m-1}-3 q+2\right), m \geq 2, \\
g_{K_{2 m}-K_{4}}(q)= & q^{m(m-1)}(q-1)\left(q^{3}-1\right) \cdots\left(q^{2 m-5}-1\right) \\
& \cdot\left(q^{4 m-10}-7 q^{2 m-6}+8 q^{2 m-7}-3 q^{2 m-8}+1\right), m \geq 3, \\
g_{K_{2 m+1}-K_{4}}(q)= & q^{m^{2}+m-4}(q-1)\left(q^{3}-1\right) \cdots\left(q^{2 m-5}-1\right) \\
& \cdot\left(q^{4 m-6}-8 q^{2 m-3}+9 q^{2 m-4}-4 q^{2 m-5}+q^{2 m-6}+4 q-3\right), \quad m \geq 2,
\end{aligned}
$$




$$
\begin{aligned}
g_{K_{2 m}-K_{5}}(q)= & q^{m(m-1)}(q-1)\left(q^{3}-1\right) \cdots\left(q^{2 m-7}-1\right) \\
& \cdot\left(q^{6 m-19}-16 q^{4 m-14}+25 q^{4 m-15}-16 q^{4 m-16}+5 q^{4 m-17}-q^{4 m-18}\right. \\
& \left.+q^{2 m-6}+11 q^{2 m-8}-15 q^{2 m-9}+6 q^{2 m-10}-1\right), m \geq 3, \\
g_{K_{2 m+1}-K_{5}}(q)= & q^{m^{2}+m-5}(q-1)\left(q^{3}-1\right) \cdots\left(q^{2 m-5}-1\right) \\
& \cdot\left(q^{4 m-9}-15 q^{2 m-5}+24 q^{2 m-6}-15 q^{2 m-7}+4 q^{2 m-8}\right. \\
& +5 q-4), m \geq 3 .
\end{aligned}
$$

To prove this conjecture for a particular value of $k$, one could try to guess a formula for $h\left(K_{n}-K_{k}, r\right)$ and then verify that it satisfies the recurrence (5.1) (with appropriate initial conditions).

For our second generalization of Theorem 4.1, we need to consider the inequivalent nondegenerate symmetric scalar products on the space $\mathbb{F}_{q}^{n}$. Standard results in orthogonal geometry over $\mathbb{F}_{q}$ (essentially equivalent to the results used in the second proof of Theorem 4.1) show that there are two such scalar products when $q$ is odd. They are defined as follows, where $a=\left(a_{1}, \ldots, a_{n}\right), b=\left(b_{1}, \ldots, b_{n}\right) \in \mathbb{F}_{q}^{n}$ :

$$
\begin{gathered}
\langle a, b\rangle_{+}=a b^{t}=\sum a_{i} b_{i} \\
\langle a, b\rangle_{-}=a D b^{t}=\alpha a_{1} b_{1}+\sum_{i=2}^{n} a_{i} b_{i} .
\end{gathered}
$$

Here, $D$ and $\alpha$ have the same meaning as in the second proof of Theorem 4.1, so, in particular, $\alpha$ is a nonsquare in $\mathbb{F}_{q}$.

Similarly, if $n$ is odd, then all nondegenerate symmetric scalar products are equivalent to (5.2) when $q$ is even. When $n=2 m$, we have (5.2) together with

$$
\langle a, b\rangle_{-}=a E b^{t}=\sum_{i=1}^{m}\left(a_{2 i-1} b_{2 i}+a_{2 i} b_{2 i-1}\right),
$$

where $E$ is defined in the second proof of Theorem 4.1.

Now, suppose $G$ is a graph with vertex set $\left\{v_{1}, \ldots, v_{n+1}\right\}$ such that $v_{n+1}$ is an apex. Let $b_{G}^{+}(q)$ (respectively, $\left.b_{G}^{-}(q)\right)$ denote the number of ordered bases $\left(u_{1}, \ldots, u_{n}\right)$ of $\mathbb{F}_{q}^{n}$ such that

$$
\left.\left\langle u_{i}, u_{j}\right\rangle_{+}=0 \text { (respectively, }\left\langle u_{i}, u_{j}\right\rangle_{-}=0\right),
$$

whenever $i \neq j$ and $i j \notin E(G)$. Such an ordered basis forms the rows of a matrix $A \in \mathrm{GL}(n, q)$ such that $\left(A H A^{t}\right)_{i j}=0$ whenever $i \neq j$ and $i j \notin E(G)$, where $H=I, D$, or $E$ depending on which of the scalar products we are considering. It follows that the second proof of Theorem 4.1 extends mutatis mutandis to give the following result.

Theorem 5.3. Let $G$ be as above. If $q$ is odd or if $q$ is even and $n$ is even, then

$$
g_{G}(q)=\frac{b_{G}^{+}(q)}{\# \Omega^{+}(n, q)}+\frac{b_{G}^{-}(q)}{\# \Omega^{-}(n, q)} .
$$


If $q$ is even and $n$ is odd, then

$$
g_{G}(q)=\frac{b_{G}^{+}(q)}{\# \Omega(n, q)} .
$$

As an example of the use of Theorem 5.3, let $K_{1, s}$ denote the star consisting of one vertex connected to $s$ other vertices, and let $G=K_{n+1}-K_{1, s}$ for $n>s+1$. In other words, $G$ consists of $K_{n+1}$ with $s$ edges removed which are incident to a common vertex. In particular, $K_{n+1}-K_{1,1}=K_{n+1}-K_{2}$, the special case $k=2$ of $K_{n}-K_{k}$ considered above (with $n$ replaced by $n+1$ ).

Theorem 5.4. We have

$$
\begin{aligned}
g_{K_{2 m-1}-K_{1, s}}(q)= & q^{m^{2}+m-s-1}(q-1)\left(q^{3}-1\right) \cdots\left(q^{2 m-3}-1\right) \\
& \cdot\left(q^{2 m}-q^{s}-q+1\right), \quad s \leq 2 m-3, \\
g_{K_{2 m}-K_{1, s}}(q)= & q^{m(m-1)}(q-1)\left(q^{3}-1\right) \cdots\left(q^{2 m-3}-1\right) \\
& \cdot\left(q^{2 m-1-s}-1\right), \quad s \leq 2 m-2 .
\end{aligned}
$$

Proof. According to Theorem 5.3, we need to count the number of ordered bases $\left(u_{1}, \ldots, u_{n}\right)$ of $\mathbb{F}_{q}^{n}$ satisfying

$$
\left\langle u_{1}, u_{2}\right\rangle_{+}=\cdots=\left\langle u_{1}, u_{s+1}\right\rangle_{+}=0,
$$

as well as the number of ordered bases $\left(u_{1}, \ldots, u_{n}\right)$ of $\mathbb{F}_{q}^{n}$ satisfying

$$
\left\langle u_{1}, u_{2}\right\rangle_{-}=\cdots=\left\langle u_{1}, u_{s+1}\right\rangle_{-}=0
$$

(except that when $q$ is even and $n$ is odd, we only have one type of scalar product). Let $u_{1}^{\perp}$ denote the set of all vectors orthogonal to $u_{1}$, with respect to whatever scalar product is under consideration. We always have $\operatorname{dim} u_{1}^{\perp}=n-1$. Once we have chosen $u_{1}$, if $u_{1} \notin u_{1}^{\perp}$, then there are $\left(q^{n-1}-1\right)\left(q^{n-1}-q\right) \cdots\left(q^{n-1}-q^{s-1}\right)$ choices for $u_{2}, \ldots, u_{s+1}$, and then $\left(q^{n}-q^{s+1}\right) \cdots\left(q^{n}-q^{n-1}\right)$ choices for $u_{s+2}, \ldots, u_{n}$. On the other hand, if $u_{1} \in u_{1}^{\perp}$, then there are $\left(q^{n-1}-q\right)\left(q^{n-1}-q^{2}\right) \cdots\left(q^{n-1}-q^{s}\right)$ choices for $u_{2}, \ldots, u_{s+1}$, and then $\left(q^{n}-q^{s+1}\right) \cdots\left(q^{n}-q^{n-1}\right)$ choices for $u_{s+2}, \ldots, u_{n}$ as before. Hence, to complete the computation, we need to know the number $N_{ \pm}(n)$ of $u_{1}$ for which $u_{1} \in u_{1}^{\perp}$, i.e, $\left\langle u_{1}, u_{1}\right\rangle_{ \pm}=0$. When $q$ is even, it is easy to compute $N_{ \pm}(n)$; when $n$ is odd, this number appears, e.g. in [4, Theorems 65 and 66] and [10, Theorems 1.26 and 1.37]. The values 
are

$$
\begin{aligned}
& N_{+}(n)=N(n)=q^{n-1}, \quad q \text { odd and } n \text { odd, } \\
& N_{+}(n)=q^{n-1}+q^{\frac{n}{2}}-q^{\frac{n}{2}-1} \text {, either } q \text { is odd and } n \equiv 0(\bmod 4) \text {, } \\
& \text { or } q \equiv 1(\bmod 4) \text { and } n \equiv 2(\bmod 4) \text {, } \\
& N_{+}(n)=q^{n-1}-q^{\frac{n}{2}}+q^{\frac{n}{2}-1} \text {, either } q \text { is odd and } n \equiv 2(\bmod 4) \text {, } \\
& \text { or } q \equiv 1(\bmod 4) \text { and } n \equiv 0(\bmod 4) \text {, } \\
& N_{-}(n)=q^{n-1}-q^{\frac{n}{2}}+q^{\frac{n}{2}-1} \text {, either } q \text { is odd and } n \equiv 0(\bmod 4) \text {, } \\
& \text { or } q \equiv 1(\bmod 4) \text { and } n \equiv 2(\bmod 4) \text {, } \\
& N_{-}(n)=q^{n-1}+q^{\frac{n}{2}}-q^{\frac{n}{2}-1} \text {, either } q \text { is odd and } n \equiv 2(\bmod 4) \text {, } \\
& \text { or } q \equiv 1(\bmod 4) \text { and } n \equiv 0(\bmod 4) \text {, } \\
& N(n)=q^{n-1}, \quad q \text { even and } n \text { odd, } \\
& N_{+}(n)=q^{n-1}, \quad q \text { even and } n \text { even, } \\
& N_{-}(n)=q^{n}, \quad q \text { even and } n \text { even. }
\end{aligned}
$$

It is now a routine computation (which we omit) to obtain the stated formulas (5.3) and (5.4).

\section{Some Related Negative Results}

In Theorem 3.3, we showed that the Kontsevich conjecture for graphs with apexes is equivalent to counting nonsingular symmetric matrices over $\mathbb{F}_{q}$ with specified "holes" (entries equal to 0 ), with no holes on the main diagonal. A related problem that comes to mind is the case of arbitrary matrices, rather than symmetric matrices. Thus, let $S$ be any subset of $[n] \times[n]$, and let $h_{S}(q)$ denote the number of matrices $A \in \operatorname{GL}(n, q)$ whose support is contained in $S$, i.e., $A_{i j}=0$ whenever $(i, j) \notin S$. For instance,

$$
h_{[n] \times[n]}(q)=\# \operatorname{GL}(n, q)=\left(q^{n}-1\right)\left(q^{n}-q\right) \cdots\left(q^{n}-q^{n-1}\right) .
$$

Question. Is the function $h_{S}(q)$ always a polynomial in $q$ ?

According to Kontsevich (private communication), a negative answer follows from the existence of the Fano plane $F$ (the projective plane of order two, with three points on a line and seven points in all). We have not been able to understand this remark of Kontsevich. However, if we take $n=7$ and let $S \subset$ [7] $\times$ [7] be the support of the incidence matrix of $F$ (so $\# S=21$ ), then Stembridge has shown that $h_{S}(q)$ is not a polynomial. More precisely,

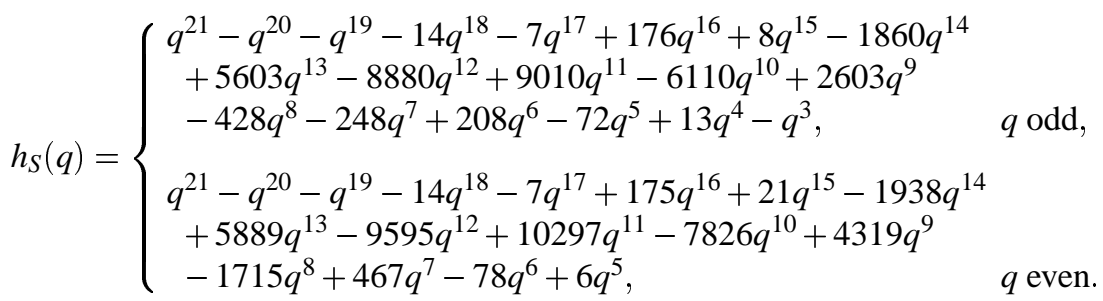


Moreover, Stembridge has also verified that $S$ is the smallest counterexample to the polynomiality of $h_{T}(q)$, in the sense that $h_{T}(q)$ is a polynomial whenever \#T $\leq 21$ or whenever $n \leq 7$, except when $T$ can be transformed to $S$ by row and column permutations.

Now, let $S$ be a symmetric subset of $[n] \times[n]$, i.e., $(i, j) \in S \Leftrightarrow(j, i) \in S$. Define $k_{S}(q)$ to be the number of invertible $n \times n$ symmetric matrices over $\mathbb{F}_{q}$ whose support is contained in $S$. Suppose $T$ is a subset of $[n] \times[n]$ for which $h_{T}(q)$ is not a polynomial. (The discussion above shows that we can take $n=7$.) Let $A$ be an $n \times n$ matrix over $\mathbb{F}_{q}$. Then $A$ is counted by $h_{T}(q)$ if and only if the $2 n \times 2 n$ matrix

$$
B=\left[\begin{array}{cc}
0 & A \\
A^{t} & 0
\end{array}\right]
$$

is a nonsingular symmetric matrix with support contained in

$$
S=\{(i, j+n),(j+n, i):(i, j) \in T\} \subset[2 n] \times[2 n] .
$$

Hence, $k_{S}(q)$ is not a polynomial. Unfortunately, all the main diagonal elements of examples of the form $\left[\begin{array}{cc}0 & A \\ A^{t} & 0\end{array}\right]$ are holes, so we cannot use Theorem 3.3 to deduce that we have a counterexample to Kontsevich's conjecture.

As pointed out by Stembridge, there are even simpler examples of symmetric sets $S \subset[n] \times[n]$ for which $k_{S}(q)$ is not a polynomial. Any symmetric matrix of odd order $n$ with 0's on the main diagonal over a field of characteristic 2 is singular. Hence, for $n$ odd, we can choose $S$ to be any subset of $[n] \times[n]$ that includes no element of the form $(i, i)$ and contains at least one transversal (i.e., a subset $(i, w(i))$ where $w$ is a permutation of $[n]$ ). (This last condition is equivalent to $k_{S}(q) \neq 0$ for some $q$.) Then $k_{S}(q)=0$ for $q=2^{m}$, but $k_{S}(q) \neq 0$ for some $q$, so $k_{S}(q)$ is not a polynomial. Since the prime 2 plays such a special role in this example, perhaps the function $h_{S}(q)$ or $k_{S}(q)$ is a polynomial in $q$ for odd $q$. A good place to look for a counterexample to this suggestion would be when $S$ is the support of a projective plane of odd order, but even for the plane of order 3 , we are unable to compute $h_{S}(q)$.

There are various natural generalizations of Kontsevich's conjecture. For instance, the spanning trees of a connected graph $G$ form the bases of the graphic matroid associated with $G$ (see, e.g., [3, 1.3.B]). Thus, if $M$ is any matroid on the set $\left\{e_{1}, \ldots, e_{s}\right\}$, then define in complete analogy to (1.1)

$$
Q_{M}(x)=\sum_{B} x^{B},
$$

where $B$ ranges over all bases of $M$. Let $g_{M}(q)$ denote the number of ways of substituting elements of $\mathbb{F}_{q}$ for the variables of $Q_{M}(x)$ such that $Q_{M}(x) \neq 0$. We can generalize Kontsevich's conjecture by asking whether $g_{M}(q)$ is always a polynomial function of $q$. There are, however, very simple counterexamples. For instance, if $M$ is the four-point line so that

$$
Q_{M}(x)=x_{1} x_{2}+x_{1} x_{3}+x_{2} x_{3}+x_{1} x_{4}+x_{2} x_{4}+x_{3} x_{4},
$$


then it can be shown that

$$
g_{M}(q)= \begin{cases}q(q-1)\left(q^{2}-1\right), & q \equiv 1(\bmod 3), \\ q(q-1)\left(q^{2}+1\right), & q \equiv 2(\bmod 3), \\ q^{3}(q-1), & q \equiv 0(\bmod 3) .\end{cases}
$$

Matroid theorists will notice that the four-point line is not a regular (or unimodular) matroid, but every graphic matroid is regular. Hence, it is natural to ask whether $g_{M}(q)$ might be a polynomial for regular matroids $M$. However, Stembridge has shown that, for the regular matroid $M$ called R10 in Oxley's book [7], $g_{M}(q)$ is not a polynomial.

Although for the four-point line $g_{M}(q)$ is not a polynomial, note that it is a quasipolynomial, i.e., for some $N>0$ (here, $N=3$ ), it is a polynomial on the different residue classes modulo $N$. Thus, it might be interesting to consider for which matroids $M$ (or for even more general varieties than the zeros of $Q_{M}(x)$ ) is $g_{M}(q)$ a quasipolynomial. In particular, if Kontsevich's conjecture is false, is it at least true that $g_{G}(q)$ is a quasipolynomial?

Acknowledgments. The author is grateful to Jeff Lagarias for calling my attention to the conjecture of Kontsevich and for providing him with a copy of his notes of Kontsevich's lecture and a related lecture of I.M. Gelfand. The author also wishes to thank Tim Chow and John Stembridge for some useful discussions.

\section{References}

1. A. Albert, Symmetric and alternating matrices in an arbitrary field, Trans. Amer. Math. Soc. 43 (1938), 386-436.

2. L. Carlitz, Representations by quadratic forms in a finite field, Duke Math. J. 21 (1954) 123-128.

3. H. Crapo, Examples and basic concepts, In: Theory of Matroids, N. White, Eds., Cambridge University Press, Cambridge, 1986.

4. L.E. Dickson, Linear Groups with an Exposition of the Galois Field Theory, B. G. Teubner, Leipzig, 1901; Dover, New York, 1958, reprint.

5. B.M. Dwork, On the rationality of the zeta function of an algebraic variety, Amer. J. Math. 82 (1960) 631-648.

6. J. MacWilliams, Orthogonal matrices over finite fields, Amer. Math. Monthly 76 (1969) $152-164$.

7. J.G. Oxley, Matroid Theory, Oxford University Press, New York, 1992.

8. R. Stanley, Enumerative Combinatorics, Vol. 2, Cambridge University Press, Cambridge, 1999.

9. J. Stembridge, Counting points on varieties over finite fields related to a conjecture of Kontsevich, Ann. Combin. 2 (1998) 365-385.

10. Z. Wan, Geometry of Classical Groups over Finite Fields, Studentlitteratur, Lund, 1993. 